\title{
Modificação do Método de Dosagem de Fosfato de Heinonen \& Lahti (1981)
}

\author{
Laíce S. Rabelo, Anelise S. Mendonça, Milton V. Coelho, \\ Saulo G. Pereira \& Hugo C. S. Melo
}

Heinonen \& Lahti (1981) $)^{1,2}$ publicaram um método colorimétrico para dosagem de fosfato inorgânico que é até hoje padrão para estudos de enzimas que realizam a hidrólise de ATP, como ATPases, por exemplo. O método consiste em uma solução de acetona P.A, ácido sulfúrico $5 \mathrm{~N}$ e molibdato de amônio $10 \mathrm{mM}$ numa proporção de 2:1:1. A aquisição de acetona P.A., mesmo para instituições de ensino e pesquisa, é controlada pela polícia federal, uma vez que pode ser utilizado no refino de cocaína. Sendo assim, este trabalho buscou analisar a substituição da acetona P.A. por álcool etílico P.A., uma vez que se trata de um reagente de fácil aquisição. A solução de álcool etílico P.A., ácido sulfúrico $5 \mathrm{~N}$ e molibdato de amônio $10 \mathrm{mM}$ na proporção de 7:2:1 foi mais sensível que a solução do método original, mostrou linearidade para concentrações de fosfato inorgânico de até $400 \mathrm{mM}$, produziu uma cor estável por cerca de 2 horas e não sofreu influência estatisticamente significante por outros reagentes utilizados em análises bioquímicas, como Triton X-100 1\%, Tween 1\%, EDTA 5mM, EGTA $5 \mathrm{mM}$, Imidazol $20 \mathrm{mM}$, Triz $20 \mathrm{mM}$, CAPS $20 \mathrm{mM}$, HEPES 18,75 $\mathrm{mM}$, BSA $0,5 \mathrm{mg} / \mathrm{mL}, \mathrm{KCl} 1 \mathrm{M}$ e NaCl 1M. Assim, o método de Heinonen e Lahti (1981) modificado para o uso de álcool etílico na proporção de 7:2:1 se mostrou capaz de substituir com eficácia o método original, proporcionando resultados tão confiáveis quanto o método original com a facilidade da compra do reagente.

Palavras-chave: fosfato inorgânico; quantificação; ATPases; colorimetria.

Heinonen \& Lahti (1981) ${ }^{1,2}$ published a colorimetric method for inorganic phosphate dosage, that still standard for studies of enzymes that perform ATP hydrolysis, such as ATPases. The method consists of using a solution of acetone P.A, $5 \mathrm{~N}$ sulfuric acid and $10 \mathrm{mM}$ ammonium molybdate in a ratio of $2: 1: 1$. The acquisition of acetone P.A., even for educational and research purposes, is controlled by the Brazilian federal police, since it can be used in cocaine refining. Thus, this work sought to analyze the substitution of acetone P.A. for ethyl alcohol P.A., since it is a readily available reagent. The solution of ethyl alcohol PA, $5 \mathrm{~N}$ sulfuric acid and $10 \mathrm{mM}$ ammonium molybdate in the 7:2:1 ratio was more sensitive than the solution of the original method, showed linearity for concentrations of inorganic phosphate of up to $400 \mathrm{mM}$, produced a stable color by about 2 hours and did not undergo statistically significant influence by other comon reagents used in biochemical analyzes such as $1 \%$ Triton X-100, $1 \%$ Tween, 5 mM EDTA, $5 \mathrm{mM}$ EGTA, $20 \mathrm{mM}$ Imidazole, $20 \mathrm{mM}$ Triz, $20 \mathrm{mM}$ CAPS, HEPES $18.75 \mathrm{mM}, 0.5 \mathrm{mg} / \mathrm{mL}$ BSA, $1 \mathrm{M} \mathrm{KCl}$, and $1 \mathrm{M} \mathrm{NaCl}$. Thus, the method of Heinonen e Lahti (1981) modified for the use of ethyl alcohol in the proportion of 7:2:1 proved capable of effectively replacing the original method, providing results as reliable as the original method with ease of purchase of the reagent.

Keywords: inorganic phosphate; quantification; ATPases; colorimetry. 


\section{Introdução}

Heinonen \& Lahti $(1981)^{1,2}$ descreveram um método simples, para a determinação de fosfato inorgânico $(\mathrm{Pi})$ e que até hoje está intimamente envolvido com pesquisas que envolvem a dosagem de Pi em sistemas biológicos, como atividade ATPásica por exemplo (Soares Melo e Coelho, 2007; Soares Melo et al., 2018; Soares Melo e Coelho, 2018) $)^{1-7}$. Este método consiste na utilização de uma solução de acetona P.A, ácido sulfúrico $5 \mathrm{~N}$ e molibdato de amônio $10 \mathrm{mM}$ numa proporção de 2:1:1 para permitir a formação do complexo fosfomolibdato e a solubilização desse complexo com acetona em meio ácido. Este complexo possui uma cor amarela brilhante que pode ser quantificada colorimetricamente em espectrofotômetro a $355 \mathrm{~nm}$, tão eficiente quanto o método de Fiske-SubbaRow(Lindberg \& Enster, 1956; Peterson, $1978)^{3,4}$, tem como vantagem a não hidrolise de ésteres de fosfato e anidridos pelo ácido durante o desenvolvimento e a medição da cor.

A obtenção de acetona com alto grau de pureza para os laboratórios de pesquisa ou análise é burocrática, uma vez que se trata de um produto sujeito ao controle e à fiscalização da Polícia Federal em quantidade acima de 2 litros por mês, por se tratar de um dos produtos utilizados no refino de cocaína (Brasil, 2018) ${ }^{1}$. Dessa forma, buscouse avaliar a substituição da acetona por álcool etílico no método de Heinonen\&Lahti $(1981)^{2}$ para dosagem de fosfato inorgânico, uma vez que a obtenção de álcool é mais simples e possui custo menor.

\section{Materiais e Métodos}

Baseado no método de Heinonen \& Lahti $(1981)^{2}$, comparou-se a solução formada pela mistura de acetona P.A. (ou propanona - $\mathrm{CH}_{3}(\mathrm{CO}) \mathrm{CH}_{3}$ ), ácido sulfúrico $\left(\mathrm{H}_{2} \mathrm{SO}_{4}\right) 5 \mathrm{~N}$ e molibdato de amônio $\left(\left(\mathrm{NH}_{4}\right)_{6} \mathrm{Mo}_{7} \mathrm{O}_{24}{ }_{4} \mathrm{H}_{2} \mathrm{O}\right)$ $10 \mathrm{mM}$ na proporção de 2:1:1 e em volume final de 3 $\mathrm{mL}$, respectivamente, com a solução em que a acetona foi substituída por álcool etílico (ou etanol - C2H5OH) P.A. em diferentes proporções. $\mathrm{O}$ conteúdo final foi misturado em vórtex. Esse método gera um complexo de cor amarela, o fosfomolibdato de amônio $\left(\left(\mathrm{NH}_{4}\right)_{3} \mathrm{PMo}_{12} \mathrm{O}_{40}\right)$, que é solubilizado por acetona em meio ácido para que possa ser quantificado colorimetricamente.
A dosagem de fosfato inorgânico gerado em sistemas biológicos, envolve a adição de ácido cítrico $1 \mathrm{M}$ ao final da reação de interesse para cessar a formação do produto e, assim, prosseguir com a dosagem conforme o método. Nesse experimento, não utilizou-se ácido cítrico, pois as dosagens foram realizadas com adição de fosfato diácido de potássio $\left(\mathrm{KH}_{2} \mathrm{PO}_{4}\right)$ em concentrações pré-definidas. Ao volume final, foram adicionado $200 \mu \mathrm{L}$ de $\mathrm{H}_{2} \mathrm{O}$ Milli-Q correspondente ao volume do ensaio biológico (amostra biológica e adenosina trifosfato).

Utilizaram-se etanol P.A., ácido sulfúrico $5 \mathrm{~N}$ e molibdato de amônio $10 \mathrm{mM}$ nas proporções de $2: 1: 1,3: 1: 1,1: 1: 1$, 4:3:1, 6:3:1 e 7:2:1, respectivamente, para comparar com a solução proposta por Heinonen \& Lahti (1981) ${ }^{2}$, em termos de sensibilidade, linearidade, estabilidade e interferência por substâncias comumente utilizadas em laboratório. Todas as dosagens foram realizadas em triplicatas e foi utilizado espectrômetro HITACHI U-2000 na frequência de $355 \mathrm{~nm}$, e cubetas de quartzo. As análises estatísticas foram procedidas com o software GraphpadPrism 8.0.2.263.

\section{Resultados e Discussão}

Segundo Heinonen \& Lahti (1981) $)^{2}$, a concentração de molibdato final deve ser alta o suficiente para formar a cor amarela instantaneamente, assim que complexado com o fosfato inorgânico. Além disso, a concentração final de ácido sulfúrico deve ser pelo menos $0,2 \mathrm{~N}$ para prevenir a cor amarela de surgir sem a presença de fosfato inorgânico. Por fim, a quantidade de acetona deve ser de, pelo menos, um terço do volume final da solução para dissolver todos componentes da mistura. Uma vez que acetona e etanol são solventes diferentes.

Dessa maneira, o presente trabalho não se constituiu apenas na substituição da acetona por etanol, e sim na avaliação de diferentes proporções dos componentes da solução para comparação da sensibilidade com o método original Figura 1.

A Figura 1 mostra que, com exceção da proporção de 1:1:1 (etanol:molibdato de amônio:ácido sulfúrico), todas as outras proporções testadas apresentaram similaridade aos resultados com acetona, com potencial de substituição, inclusive a proporção de 7:2:1 foi mais sensível ao fosfato inorgânico que a solução de acetona. 


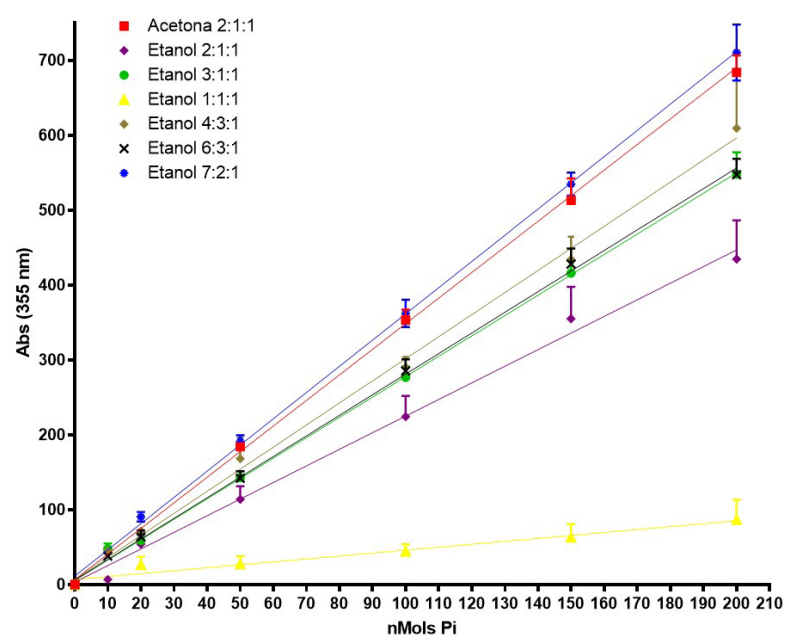

Figura 1: Curva de dosagem de Pi com acetona e etanol em diferentes proporções. Todos os pontos de dosagem de $\mathrm{Pi}$, com a respectiva proporção da solução de dosagem, foram realizados em triplicatas, agitados em vórtex e analisadas com cubetas de quartzo em espectrofotômetro.

Diferente do método original com acetona, a utilização de etanol na proporção de 7:2:1 foi linear para dosagem de Pi de $50 \mathrm{nM}$ somente até cerca de $400 \mathrm{nM}$, apresentando coeficiente de linearidade (R2) de 0,9786 (Figura 2). Apesar de o método com acetona apresentar linearidade até cerca de $1200 \mathrm{nM}$ de Pi, o método com etanol com mais de $400 \mathrm{nM}$ de Pia presentou uma queda do coeficiente de linearidade para 0,8065 , apresentando então uma estabilização não proporcional da cor até 1500 nM de Pi.

Nas duas situações do método, com acetona ou etanol, o aparecimento da cor se desenvolve imediatamente após a mistura dos reagentes (cerca de 15s). No entanto, o método original com acetona se manteve estável por até 6 horas, enquanto que, com a utilização de álcool a estabilidade foi mantida por pouco mais de 2 horas sem perda significativa da cor do complexo (Figura 3). Ainda que haja uma diferença clara na estabilidade da cor, 2 horas continua sendo suficiente para a medição da maioria dos testes em espectrofotômetro.

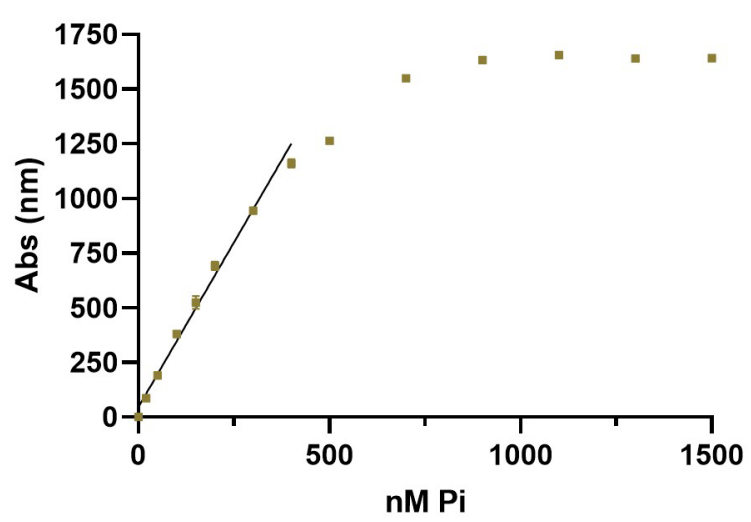

Figura 2: Linearidade da intensidade de cor do fosfomolibdato. Em todos os pontos de dosagem de Pi foram utilizados etanol, molibdato de amônio e ácido sulfúrico na proporção de 7:2:1, foram feitos em triplicatas, agitados em vórtex e analisadas com cubetas de quartzo em espectrofotômetro. A linha mostra a faixa de linearidade até $400 \mathrm{nM}$ de $\mathrm{Pi}$, com coeficiente de linearidade de 0,9830 e a equação da reta $\mathrm{Y}$ $=3,501 * \mathrm{X}+11,54$

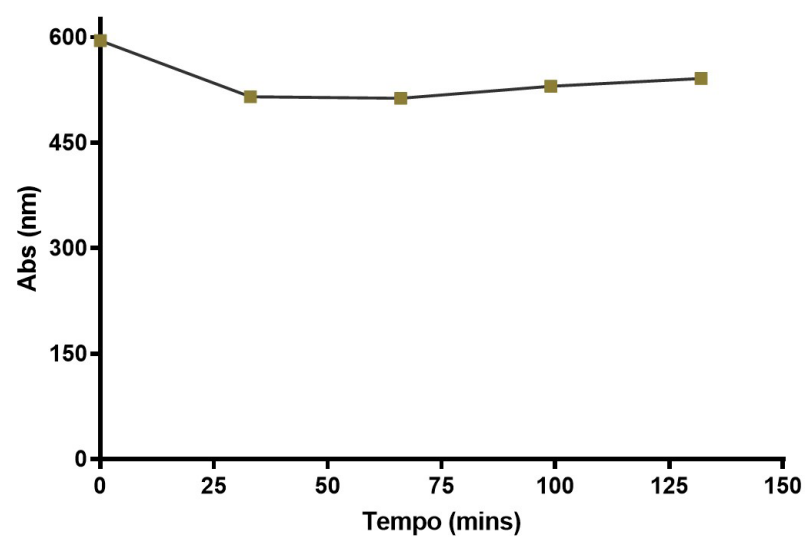

Figura 3: Estabilidade da cor do fosfomolibdato. $100 \mathrm{nMols}$ de fosfato diácido de potássio (KH2PO4) foram dosados com etanol, molibdato de amônio e ácido sulfúrico na proporção de 7:2:1, em triplicatas, agitados em vórtex e analisadas com cubetas de quartzo em espectrofotômetro.

Foi também avaliada a interferência de diversas substâncias comumente utilizadas em bioquímicano método de dosagem de Pi de Heinonen \& Lahti (1981)², substituindo acetona por álcool. Compostos que complexam 
com molibdato, como Pirofosfato (PPi) e ATP, podem atrasar a formação da cor, porém na concentração final de molibdato utilizada no método modificado não apresentou diferença estatística significante $(\mathrm{p}<0,05)$.

Outras substâncias também não demonstraram alterações estatisticamente significantes em relação ao controle. Foram realizados os testes de Análise de Variância tradicional (ANOVA) e Kruskal-Wallis, e em nenhum deles foi verificada diferença estatisticamente significante entre o controle e as substancias de interferência, como Triton X-100 1\%, Tween 1\%, EDTA 5mM, EGTA 5 mM, Imidazol $20 \mathrm{mM}$, Triz $20 \mathrm{mM}$, CAPS $20 \mathrm{mM}$, HEPES $18,75 \mathrm{mM}$, BSA $0,5 \mathrm{mg} / \mathrm{mL}, \mathrm{KCl} 1 \mathrm{M}$ e $\mathrm{NaCl} 1 \mathrm{M}$ ((Figura 4).

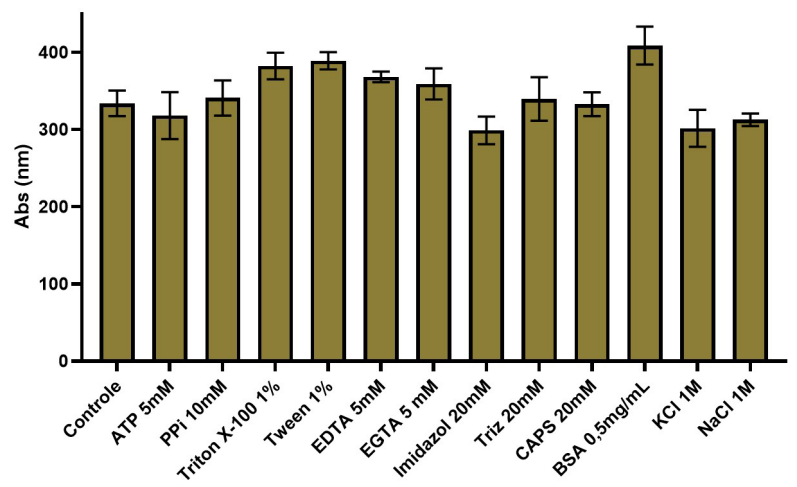

Figura 4: Análise da interferência de alguns reagentes na cor do fosfomolibdato. $100 \mathrm{nMols}$ de fosfato diácido de potássio (KH2PO4) foram adicionados aos tubos com os reagentes nas concentrações indicadas, e no volume final de $200 \mu \mathrm{L}$ acertado com H2O Milli-Q. Em seguida, foram adicionados $2 \mathrm{~mL}$ da solução de dosagem (Etanol P.A., Molibidato de amônio $10 \mathrm{mM}$ e Ác. Sulfúrico $5 \mathrm{~N}$ na proporção 7:2:1) ao tubo e foi agitado vigorosamente por 15 segundos. $200 \mu \mathrm{L}$ de Ác. Cítrico $1 \mathrm{M}$ foram adicionados na sequência com mais 10 segundos de vigorosa agitação. Os ensaios foram analisados a $355 \mathrm{~nm}$ em espectrofotômetro.

Similar ao método original, compostos que absorvem luz no comprimento de luz entre 390-420nm (como vitaminas, flavoproteínas, hemoproteínas, interferem nos resultados do teste, porém esta interferência pode ser diminuída pela diminuição da concentração dessas substâncias ou pela precipitação das mesmas a priori da dosagem de Pi (Heinonen \& Lahti, 1981)2.

\section{Considerações Finais}

O método de dosagem de fosfato inorgânico de Heinonen \& Lahti $(1981)^{2}$ é comumente utilizado por laboratórios de todo mundo, especialmente, quando se trata do estudo de ATPases e sua atividade enzimática. A aquisição de acetona para realização do método é burocrática, e nem sempre os laboratórios podem permitir-se a espera da compra. Assim, a substituição da acetona por álcool etílico é uma opção de baixo custo e fácil aquisição para laboratórios, além de evitar que a pesquisa tenha atrasos desnecessários enquanto espera a compra do material em questão. O método modificado neste trabalho mostrou uma excelente sensibilidade, uma boa estabilidade da cor e não sofre interferência por vários reagentes comumente utilizados em laboratórios de bioquímica, apontando que a solução de etanol, molibdato de amônio e ácido sulfúrico na proporção de 7:2:1 podem substituir de forma eficaz a solução proposta pelo método original de Heinonen\&Lahti (1981)2, sem prejuízos para a análise.

\section{Suporte}

Esta pesquisa foi desenvolvida com suporte da Universidade Federal de Uberlandia (UFU), do Conselho Nacional de Desenvolvimento Científico e Tecnológico $(\mathrm{CNPq})$ e da Fundação de Amparo à Pesquisa do Estado de Minas Gerais (FAPEMIG).

\section{Referências}

1. BRASIL. Ministério da Segurança Pública. Gabinete do Ministro. Portaria $n^{\circ} 256$, de 26 de dezembro de 2018. Estabelece procedimentos para o controle e a fiscalização de produtos químicos e define os produtos químicos sujeitos a controle pela Polícia Federal. Diário Oficial da União, Brasília, DF, 27 dez. 2018. Seção 1, p. 169.

2. Heinonen, J. K.; Lahti, R. J. Anal Biochem. 1981, 113, 313.

3. Lindberg, O.; Enster, L. MethodsBiochem. Anal. 1956, 3, 1.

4. Peterson, G. L. Anal Biochem. 1978, 84, 1, 164.

5. Soares Melo, H. C.; Coelho, M. V. Acta Biochim Pol. 2007, 54, 3, 575.

6. Soares Melo, H. C.; Coelho, M. V. Revista NBC. 2018, 8, 15, 70.

7. Soares Melo, H. C.; Cruz, G. C. N. D.; Sousa, M. V. D.; Coelho, M. V. InternationalJournalofRecentScientificResearch. 2018, 9, 8, 28417. 


\title{
Laíce S. Rabelo', Anelise
}

\section{S. Mendonça', Milton V.}

Coelho', Saulo G. Pereira² \&

Hugo C. S. Melo* 2

\author{
${ }^{1}$ Universidade Federal de Uberlândia (UFU), Instituto de Genética \\ e Bioquímica (INGEB). Av. Para, 1720 - Campus Umuarama - \\ Bloco 2E - Sala 39b - Uberlândia - MG - CEP 38405-320 \\ ${ }^{2}$ Faculdade Patos de Minas. Av. Juscelino Kubitschek de Oliveira, \\ $\mathrm{n}^{\circ} 1220$, Bairro Cristo Redentor, Patos de Minas, MG, Brasil - \\ CEP 38706-401.
}

*E-mail: hugo.some@gmail.com 